\title{
A FORMAÇÃO DE PROFISSIONAIS DA SAÚDE: APRENDIZAGEM SIGNIFICATIVA À LUZ DA PROMOÇÃO DA SAÚDE
}

\author{
Anna Maria Chiesa ${ }^{1}$, Débora Dupas Gonçalves do Nascimento ${ }^{2}$, Luzmarina Aparecida Doretto Braccialli ${ }^{3}$, \\ Maria Amélia Campos de Oliveira ${ }^{4}$, Maria Helena Trench Ciampone ${ }^{5}$
}

RESUMO: Discute-se a formação dos profissionais de saúde na perspectiva da implementação das Diretrizes Curriculares Nacionais (DCN), com enfoque na promoção da saúde. Toma-se como eixo da formação o empowerment, que leva ao desenvolvimento de competências. A integração ensino-serviço e a utilização de metodologias ativas de ensino são apontadas como estratégias para a formação voltada para o mundo do trabalho e para as necessidades da população, visando o aprendizado significativo.

PALAVRAS-CHAVE: Promoção da saúde; Aprendizagem; Educação baseada em competência; Prática profissional.

\section{HEALTH PROFESSIONALS' EDUCATION: A SIGNIFICANT LEARNING GUIDED BY HEALTH PROMOTION}

ABSTRACT: This article discusses health professionals' education oriented to the implementation of the National Curricular Guidelines by focusing on health promotion. Empowerment is the axis which enables the development of competences. In-service education and active teaching methods are also pointed out as strategies to develop a professional education related to the work process and to the population's health needs, aiming a meaningful learning experience.

KEYWORDS: Health promotion; Learning; Competency-baed education; Professional practice.

\section{LA EDUCACIÓN DE LOS PROFESIONALES DE SALUD: EL APRENDIZAJE SIGNIFICATIVO DIRIGIDO POR LA PROMOCIÓN DE LASALUD}

RESUMEN: Se discute la formación de los profesionales de salud en la perspectiva de la implementación de las Directrices Curriculares Nacionales (DCM), con enfoque en la promoción de la salud. Se toma como eje de la formación el empowerment, que lleva al desarrollo de competencias. La integración de la enseñanza y del servicio y la utilización de metodologías activas de enseñanza son señaladas como estrategias para la formación hacia el mundo del trabajo y para las necesidades de salud de la población, con la finalidad de llegar a un aprendizaje significativo.

PALABRAS CLAVE: Promoción de la salud; Aprendizaje; Educación basada en competencias; Práctica profesional.

\footnotetext{
${ }^{1}$ Professora. Livre-docente do Departamento de Enfermagem em Saúde Coletiva da Escola de Enfermagem da Universidade de São Paulo - USP.

${ }^{2}$ Mestranda do Programa do Pós-Graduação em Enfermagem- área de concentração Enfermagem em Saúde Coletiva da Escola de Enfermagem da USP. Preceptora da Residência Multiprofissional em Saúde da Família da Casa de Saúde Santa Marcelina/Faculdade Santa Marcelina/ Ministério da Saúde. Docente do Centro Universitário São Camilo.

${ }^{3}$ Doutoranda do Programa do Pós-Graduação em Enfermagem da Escola de Enfermagem da USP. Docente da Faculdade de Medicina de Marília FAMEMA.

${ }^{4}$ Professora Livre-docente do Departamento de Enfermagem em Saúde Coletiva da Escola de Enfermagem da USP

${ }^{5}$ Professora Livre-Docente do Departamento de Orientação Profissional da Escola de Enfermagem da USP. Doutora em Psicologia Social.
}

Autor correspondente:
Anna Maria Chiesa
Av. Dr. Enéas de Carvalho Aguiar, 419 - 05403-000 - São Paulo-SP
E-mail: amchiesa@usp.br
Recebido em: 02/03/07 Aprovado em: 23/05/07

Cogitare Enferm. 2007 Abr/Jun; 12(2):236-40 


\section{O DESAFIO DE FORMAR PROFISSIONAIS GENERALISTAS}

A promoção da saúde, um dos eixos estruturantes do Sistema Único de Saúde (SUS), diz respeito ao fortalecimento da capacidade de indivíduos e grupos sociais para intervir nos determinantes do seu processo saúde-doença. A promoção envolve cinco eixos de atuação: elaboração e implementação de políticas públicas saudáveis, criação de ambientes favoráveis à saúde, reforço da ação comunitária, desenvolvimento de habilidades pessoais, reorientação dos sistemas e dos serviços de saúde ${ }^{(1,2)}$.

Fundada em uma concepção ampliada do processo saúde-doença e seus determinantes, a promoção da saúde busca articular saberes técnicos e populares, mobilizar recursos institucionais e comunitários, públicos e privados, de diversos setores, para o enfrentamento e a resolução dos problemas de saúde. Nessa concepção, a população assume um papel ativo no processo de construção das práticas sanitárias para o enfrentamento de diversos problemas e necessidades de saúde.

Como movimento da Reforma Sanitária brasileira, ao longo da década de 80, a formulação do SUS na Constituição de 1988 e sua implantação nas duas últimas décadas, o sistema de saúde no Brasil chegou ao século XXI organizado em torno da promoção e da vigilância à saúde. Profissionais de saúde e população passam a ser vistos como sujeitos do processo e a doença deixa de ser o foco de observação, que se volta para o modo de vida e as condições de trabalho dos indivíduos e grupos sociais, e suas repercussões no processo saúde-doença.

A operacionalização dos princípios e diretrizes do SUS em um novo modelo assistencial requer novos perfis profissionais. A formação do profissional generalista requerido pelo SUS atualmente é regida pela Lei de Diretrizes e Bases (LDB) que propõe, dentre outras medidas, a substituição dos currículos mínimos pelas Diretrizes Curriculares Nacionais (DCN) para os cursos de graduação em saúde ${ }^{*(3)}$. As DCN reafirmam a necessidade e o dever das Instituições de Ensino Superior (IES) em formar profissionais de saúde voltados para o SUS, com a finalidade de adequar a formação em saúde às necessidades de saúde da população brasileira.

\footnotetext{
* Biomedicina, ciências biológicas, educação física, enfermagem, farmácia, fisioterapia, fonoaudiologia, medicina, medicina veterinária, nutrição, odontologia, psicologia, serviço social e terapia ocupacional.
}

O crescente desafio das instituições formadoras é preparar profissionais para atuar nos diferentes níveis do Sistema de Saúde, especialmente na Atenção Básica, conforme preconizam as DCN, ao postular que a formação do profissional de saúde deve estar em consonância com o sistema de saúde vigente no país, o trabalho em equipe e a atenção integral à saúde ${ }^{(3)}$.

A formação do generalista requer a inserção precoce aluno no mundo do trabalho e a sua atuação crítica e reflexiva para o desenvolvimento de uma visão global, integrada e crítica da saúde, tendo como eixo central a promoção da saúde. Currículos orientados para o desenvolvimento das competências requeridas para o trabalho em saúde no SUS devem prever oportunidades pedagógicas que assegurem aos estudantes aplicar os conhecimentos teóricos e desenvolver habilidades não apenas técnicas, mas também políticas e relacionais.

Mudanças envolvem pessoas, valores, culturas e, especificamente no campo da saúde e da educação, envolvem também questões ideológicas, sociais, econômicas e históricas. Isso significa romper com "antigos paradigmas", sem negar, entretanto, a historicidade das profissões, o acúmulo de conhecimentos e os modelos de atenção à saúde existentes no país. As mudanças na formação exigem ainda novos desenhos curriculares focados em metodologias ativas de ensino e abordagem multidisciplinar fundamentada nas ciências humanas, sociais e biológicas.

A formação inicial dos profissionais de saúde, de um modo geral, não os prepara para atuar no campo da promoção à saúde, devido ao enfoque ainda predomenantemente biologicista, curativo, médicocentrado e desarticulado das práticas em saúde. Estudos têm evidenciado o distanciamento existente entre o ensino proposto pelas escolas e as necessidades de saúde da população. A formação no campo da saúde encontra-se em crise, o ensino é descontextualizado, com ênfase nos conteúdos, sendo esses operacionalizados de uma forma tradicional. Para aproximar a formação profissional das necessidades de saúde da população é necessário superar o paradigma "conteudista" predominante.

O desafio de formar generalistas passa pela necessidade de desenvolver novas concepções do processo saúde-doença, educação, ser humano e sociedade, e novas práticas de saúde, mais horizontalizadas e centradas nos processos de trabalho 
em saúde. Estimular a compreensão dos determinantes do processo saúde-doença e trabalhar a formação profissional a partir dos eixos da promoção da saúde, valendo-se de metodologias que envolvam ativamente os sujeitos do processo ensino-aprendizagem aplicadas a situações reais e simuladas, contribui para a atuação contextualizada, crítica e participativa do futuro profissional, voltada para a intervenção nesses determinantes, o estímulo à participação popular e o controle social.

A promoção da saúde deverá ser incorporada por meio dos conceitos de empowerment, intersetorialidade, eqüidade, participação popular e autonomia, entre outros, que subsidiarão a formação profissional. Neste texto, discute-se especificamente a contribuição do empowerment para a formação em saúde.

\section{CONTRIBUIÇÕES DO EMPOWERMENT NO PROCESSO DE ENSINO-APRENDIZAGEM POR COMPETENNCIA}

A promoção da saúde possibilita que indivíduos e coletividades aumentem o controle sobre os determinantes de saúde, como emprego, renda, educação, cultura, lazer e hábitos de vida, e as conseqüentes repercussões sobre as formas de adoecer e morrer. Dessa forma, instrumentaliza a intervenção mais eficaz e efetiva sobre os mesmos. Nessa perspectiva, cabe ao Estado a responsabilidade de reduzir as diferenças, assegurar a igualdade de oportunidades e promover os meios que permitam a todos desenvolver um melhor controle sobre o viver, adoecer e morrer.

A categoria empowerment é um dos eixos centrais da promoção da saúde. Compreende desde uma visão de autonomia e até as relações de poder entre as pessoas. Vem sendo aplicada em diferentes campos de conhecimento, sofrendo alterações ao longo do tempo.

Na década de 70, essa categoria foi influenciada pelos movimentos de auto-ajuda; nos anos 80 recebeu influências da psicologia comunitária e, nos anos 90, recebeu aportes de movimentos sociais que buscavam afirmar o direito da cidadania em distintas esferas da vida social, entre as quais a prática médica, a educação em saúde e o meio ambiente ${ }^{(4)}$.

No modelo tradicional de educação, o empowerment apresenta-se sob a forma de habilidades pessoais, envolvidas na construção do conhecimento e transmitidas por aqueles que detêm o saber.

No campo da promoção da saúde e no novo paradigma da formação dos profissionais, o empowerment visa contribuir para o fortalecimento dos sujeitos envolvidos, permitindo-lhes visualizar e enfrentar os determinantes do processo saúde-doença sob uma nova visão de mundo. Profissionais de saúde e população são sujeitos do mesmo processo, pelo qual se estimula o fortalecimento de cada pessoa, individualmente e em grupos organizados.

Nessa concepção, o trabalho em grupo assume especial relevância. A abordagem grupal ainda é pouco utilizada nos serviços de saúde, e estes ainda se mantêm sob forte influência da assistência curativa e individualizada, pois é o modelo que muitos profissionais aprenderam na escola. Um novo modelo pressupõe a horizontalização do poder, necessária e adequada para trabalhar os conceitos da promoção da saúde, principalmente o empowerment, estimulando a autonomia e a construção de vínculos significativos em todas as relações humanas, e possibilitando a visualização "macro" da sociedade a partir da representação no "microgrupo".

As intervenções grupais possuem um caráter potencializador, cooperativo e oportuno quanto à possibilidade de produzir e gerir conhecimentos direcionados à introdução de mudanças concretas nos micro-espaços de trabalho das instituições de saúde. Isso pode se dar a partir do exercício do pensamento e da ação crítica, aderentes a um projeto ético-político que avance na direção de uma sociedade que qualifique ao máximo a vida, permitindo o usofruto de bens pela maioria dos cidadãos.

Para que um grupo seja efetivo em promover mudanças e transformações, ele precisa ser planejado, ter objetivos determinados e estabelecer um contrato entre os participantes e seu coordenador. Este atua como mediador do grupo, dentro de um ambiente agradável e empático, respeitoso e ético, possibilitando a exposição e discussão entre todos, a partir de diferentes percepções e vivências. A competência para trabalhar grupos nesta concepção precisa ser estimulada e desenvolvida já na formação dos profissionais, visando o desenvolvimento de solidariedade e a empatia e respeito às diferenças, como alternativas de superação de todas as formas de exclusão, com vistas à justiça e à cidadania.

Os grupos podem ter uma relação muito forte com a promoção da saúde por meio da categoria empowerment, que horizontaliza as relações de poder e 
possibilita a construção de competências e habilidades para a vida em sociedade. As instituições formadoras têm um papel fundamental para o desenvolvimento de tais competências. O ensino mais igualitário, descentralizado, no qual o professor assume um papel de mediador do processo ensino-aprendizagem, possibilita que as diferenças pessoais sejam trabalhadas, levando em consideração o contexto de vida dos sujeitos envolvidos, em busca da eqüidade de cuidado, acesso e cidadania. Um ambiente escolar estimulante e acolhedor é essencial para o desenvolvimento de relações interpessoais e atividades que proporcionem maior autonomia e visão crítica, de modo a possibilitar um aprendizado significativo, em consonância com a promoção da saúde.

Para possibilitar que indivíduos e grupos sob seus cuidados enfrentem ativamente suas necessidades e problemas de saúde, os profissionais devem desempenhar o papel de educadores, apoiando e estimulando indivíduos e grupos sociais, para que se tornem agentes da promoção e da proteção de sua saúde. Para tanto, devem desencadear um processo ensino-aprendizagem no qual o ensinar e o aprender possam se efetivar de uma forma mais horizontal e não hierárquica.

Nesse sentido, as pessoas precisam ser fortalecidas, "empoderadas" como sujeitos capazes de intervir mais em suas próprias vidas e capazes de identificar condições que afetam sua saúde, aumentando assim suas possibilidades de controle sobre os determinantes de suas condições de vida e saúde. Essa realidade é possível se fundada na compreensão da emancipação humana por meio do desenvolvimento do pensamento crítico e de ações que superem antigos paradigmas, bem como estruturas institucionais e ideológicas de opressão.

A categoria empowerment possibilita o desenvolvendo da consciência crítica e da capacidade de intervenção sobre a realidade, transformando-se, neste contexto, em um ato político libertador que se contrapõe à concepção bancária de educação. Adotado como concepção pedagógica, o empowerment eleva os indivíduos e grupos mais excluídos da sociedade à condição de cidadãos portadores de direitos.

Uma aprendizagem significativa deve estar engajada no desenvolvimento de políticas públicas saudáveis e estimular a criação de ambientes favorecedores da saúde, da mudança de comportamentos que permitam a proteção do meio ambiente, a conservação de recursos naturais e o envolvimento cada vez maior da população em projetos de promoção da saúde ${ }^{(5)}$.

A compreensão da educação como um processo dialógico, problematizador e inclusivo, que visa à construção da consciência crítica sobre ser e estar no mundo, deu origem a várias tentativas de mudanças pedagógicas em diversos países e cidades do Brasil. Essas iniciativas, em geral, estão orientadas para a elaboração de currículos integrados, orientados por competências, articulados aos diversos setores da sociedade e desenvolvidos em ambientes propícios ao aprendizado.

O foco central dessas mudanças curriculares é a maior valorização dos sujeitos envolvidos no processo ensino-aprendizagem, sejam eles docentes, estudantes ou profissionais de serviços de saúde onde ocorrem as atividades de ensino prático e o estágio curricular supervisionado. Cada um pode contribuir de maneira individualizada, muitas vezes explicitando diferenças, mas sempre na busca pedagógica de trabalhar com os estudantes as suas potencialidades no processo ensino-aprendizagem. A escola estimula o aluno a buscar e construir de maneira autônoma seu processo de aprendizagem, voltado para a realidade em que se insere, visando o desenvolvimento das competências necessárias ao trabalho em saúde.

\section{A FORMAÇÃO POR COMPETÊNCIAS}

Competência é a capacidade de utilizar diferentes recursos para solucionar com pertinência e eficácia uma série de situações ${ }^{(6-9)}$, mobilizando recursos cognitivos, afetivos e psicomotores.

Nos anos 60 e 70, a concepção de competência que influenciou a organização curricular tinha fortes raízes na linha comportamental (behaviorismo). Essa influência ainda é marcante na formação dos profissionais, principalmente da área da saúde.

A partir dos anos 80, uma concepção mais ampliada passou a ser utilizada, a competência dialógica, entendida como o desenvolvimento de atributos cognitivos, psicomotores e afetivos que conjuntamente demonstram diferentes modos de realizar com excelência uma tarefa ou um trabalho profissional. Essa concepção considera a historicidade das sociedades e das pessoas que nela se inserem, em um dado momento histórico, observando o processo de transformação dos saberes, assim como os modos de produção e reprodução social que influenciam as respostas e os atributos esperados para determinada área profissional. 
A contextualização e a construção de significados durante o processo de aprendizagem, integrando teoria e prática, possibilita a reflexão e a teorização a partir da realidade prática concreta, com o desenvolvimento de capacidades profissionais, deslocando a atenção dos conteúdos e das disciplinas até então presentes em muitos currículos.

Em um currículo orientado por competência, os conteúdos só têm significado quando emergem da prática e a partir daí passam a ser explorados com consistência e funcionalidade para o enfrentamento de situações complexas e reais, construídas socialmente. O que importa não é a transmissão do conhecimento acumulado, mas sim a possibilidade de ação, a capacidade de recorrer ao que se sabe para realizar o que se deseja, o que se projeta.

Para que a integração teoria-prática aconteça de maneira efetiva, é necessário que todos os envolvidos na formação (docentes, alunos e profissionais do serviço) entendam e vivam a horizontalização dos saberes, cada um desenvolvendo seu trabalho de modo a articular conhecimentos complementares, desenvolver as habilidades técnicas e políticas, visando atender as necessidades da população, sempre refletindo sobre suas práticas e avaliando todo o processo.

$\mathrm{Na}$ abordagem dialógica, a construção de competências e sua tradução curricular mostra-se uma opção consistente e estratégica para a formação de profissionais de saúde orientada para as necessidades sociais. No entanto, ainda há um longo caminho a ser trilhado e contradições a serem superadas, em que pese a contribuição das atuais políticas públicas de saúde e educação que oportunizam iniciativas de mudança na formação dos profissionais da saúde ${ }^{(10)}$.

\section{CONSIDERAÇÕES FINAIS}

As atuais políticas nacionais de saúde e de educação apontam para a necessidade de mudanças nos processos de formação profissional e têm estimulado e apoiado iniciativas para ampliar a responsabilidade social e a pactuação de propostas para a definição de competências e para o desenvolvimento das diretrizes curriculares nacionais.

Para fazer face aos enfrentamentos colocados pelo mundo do trabalho, essa mudança paradigmática envolvendo as concepções de saúde e educação precisa ser vivenciada durante a formação, ampliando as possibilidades de horizontalização e democratização do conhecimento. Competências e habilidades também precisam ser desenvolvidas pelos profissionais de saúde, para que se constituam como sujeitos comprometidos com a busca de equidade do cuidado, do acesso e da cidadania.

\section{REFERÊNCIAS}

1 Ministério da Saúde (BR). Secretaria de Políticas de Saúde. Projeto Promoção da Saúde. Promoção da Saúde: Declaração de Alma-Ata, Carta de Otawa, Declaração de Adelaide, Declaração de Sundsvall, Declaração de Santafé e Bogotá, Declaração de Jacarta, Rede de Megapaíses, Declaração do México. Brasília; 2001.

2 Chiesa AM. A promoção da saúde como eixo estruturante do Programa de Saúde da Família. In: Tema do $1^{\circ}$ Seminário Estadual "O Enfermeiro no Programa de Saúde da Família"; 2000 nov. 9-15; São Paulo, SP. São Paulo: Secretaria de Estado da Saúde; 2000. p. 1-7.

3 Almeida M, Maranhão E. Diretrizes curriculares nacionais para os cursos universitários da área da saúde. Londrina: Rede UNIDA; 2003.

4 Carvalho SR. Os múltiplos sentidos da categoria "empowerment" no projeto de promoção à saúde. Cad Saúde Publ. 2004;20(4):1088-95.

5 Davine MC. Currículo integrado. In: Ministério da Saúde (BR). Coordenação Geral de Desenvolvimento de Recursos Humanos para o SUS. Capacitação pedagógica para instrutor/supervisor: área da saúde. Brasília; 1994. p.39-55.

6 Hager P, Gonczi A. What is competence. Med Teach. 1996;18(1):15-8.

7 Hernández D. Políticas de certificación de competencias en América Latina. Bol Cinterfor 2002; (152):31-49. Disponível em: http://www.cinterfor.org.uy/public/ spanish/region/ampro/cinterfor/publ/boletin/152/pdf/ hern.pdf. (5 Fev 2007).

8 Perrenoud P. Construir as competências desde a escola. Porto Alegre: Artmed; 1999.

9 Ramos MN. A pedagogia das competências: autonomia ou adaptação? São Paulo: Cortez; 2001.

10 Lima VV. Competência: distintas abordagens e implicações na formação de profissionais de saúde. Interface Comun Saúde Educ. 2005;9(17):369-79. 